\title{
ILSI Europe workshop on Diet and Physical Activity - Interactions for Health: summary and conclusions
}

\author{
22- 24 March, 1999 in Chamonix, France \\ Carmen Pérez Rodrigo ${ }^{1}$, Emmanuel Van Praagh ${ }^{2}$, Michael Gibney ${ }^{3}$ and \\ Michael Siöström ${ }^{4,5}$ \\ ${ }^{1}$ Community Nutrition Unit, Department of Public Health, Luis Brinas, 18: 4 planta, E - 48013 Bilbao: \\ ${ }^{2}$ Université Blaise Pascal, UFR STAPS, BP 104, F - 63172 Aubiere: ${ }^{3}$ Department of Clinical Medicine, \\ University of Dublin, Trinity College, The Trinity Centre for Health Sciences, St. James Hospital, James' Street, \\ IRL - Dublin 8: ${ }^{4}$ Karolinska Institute, Department of Biosciences, Unit for Preventive Nutrition, Novum, \\ S - 14157 Huddinge: ${ }^{5}$ University of Örebro, Department of Physical Education and Health, S-70182 Örebro
}

\section{Introduction}

This workshop examined the links between diet and physical activity and their combined impact on health. Diet and lack of physical activity have both been linked to a number of diseases and conditions, such as cardioand cerebrovascular diseases including high blood pressure, high blood lipids, ischemic heart disease and stroke, obesity, diabetes, osteoporosis, cancer and impaired cognitive function. The present workshop was initiated by the ILSI Europe Healthy Lifestyles Task Force and convened in Chamonix, France on 22-24 March, 1999, to discuss and evaluate the science base of interactions between diet and physical activity on health.

In his introduction, the overall chair, Prof. M. Sjöström (Karolinska Institute, University of Örebro, S) summarized data showing that life-style related, non-communicable diseases already are the leading causes for years of life lost in the world ${ }^{1}$. In Europe, the top health problems share common risk factors, such as unhealthy nutrition and lack of physical exercise. An urgent need for additional basic research for a more detailed understanding of the interaction for health between diet and physical activity was acknowledged. Better methods for assessment of nutrition and physical activity in the population have to be developed, and more and better baseline data have to be collected. A better understanding of how to communicate the messages to the public and increased and more efficient action in the field of diet and physical activity interventions are needed. Effective population based strategies require people trained and competent in the discipline of public health nutrition.

Dr. B. Danse (ILSI Europe, B) presented the scope of the problem. He also referred to the ILSI Europe Concise Monograph on Healthy Lifestyles - Diet and Physical Activity that was developed in response to the European Community Action on Health Promotion, Information, Education and Training of the European Commission (EC) Directorate General V (DGV). Prof.
A. Kafatos (University of Crete, GR) summarised the activities involved in the EC Concerted Action Programme on "Nutrition and Diet for Healthy Lifestyles in Europe" which aims to build a framework for developing dietary guidelines in European countries. A conference at Crete in May 2000 will be the culmination of the project. The final report and recommendations will be presented to the European Commission, the Council of Ministers and the European Parliament.

In order to set the scene for the summary of the workshop it is helpful to note some definitions concerning Physical Activity, adopted during a Consensus Conference in Toronto ${ }^{2}$, and Public Health Nutrition, adopted by the Working Group responsible for the development of a European Master's Programme in Public Health Nutrition.

Physical activity is any body movement produced by the skeletal muscles that results in a substantial increase in energy expenditure over the resting energy expenditure. It thus includes not only exercise (undertaken with the deliberate intent of improving health or physical performance) and sport, but also equivalent energy expenditure in other types of active leisure, occupational work, and domestic chores.

Exercise is every planned, structured, and repetitive body movement done to improve or maintain one or more components of physical fitness.

Physical fitness is a human condition with physical, social and psychological dimensions, each characterised on a continuum with positive and negative poles. Positive health is associated with a capacity to enjoy life and to withstand challenges: it is not merely the absence of disease. Negative health is associated with disease and adverse health effects, and in the extreme, with premature mortality.

Public health nutrition focuses on the promotion of good health through nutrition and physical activity and the prevention of related illness in the population. 


\section{Executive summaries of the presentations}

The workshop began with an overview of nutrition and physical activity across European countries by Prof. M. Gibney (Trinity College, IRL). Patterns of intake of fatty acids show a North-South divide. Diets of countries such as Finland, Germany and the Netherlands contain a higher proportion of saturated than of monounsaturated fatty acids, whereas the opposite is true in countries such as Greece, Spain and Portugal ${ }^{3}$. Interestingly, the observed proportions do not vary between high-fat eaters and low-fat eaters. A survey of European consumer attitudes toward physical activity showed a high level of ignorance of the adverse effects of obesity and the health benefits of physical activity ${ }^{4}$. Consumers believe weight loss is necessary for physical activity to be beneficial. They are complacent about their present level of physical activity - better described as a sedentary lifestyle. A policy aimed at convincing people to increase their general level of physical activity should separate the idea of weight control from the message to increase physical activity. As consumers frequently cited relieving stress as a reason to participate in physical activity, this argument would probably be more effective than a weight control argument.

Dr. M. van Baak (Maastricht University, NL) addressed the problem of obesity as an imbalance between energy intake and energy expenditure. An increase in the prevalence of obesity, observed in many parts of the world, has been linked with declines in the level of daily physical activity as well as changing food habits. The level of physical activity - a function of intensity and duration of the activities performed - is the most variable component of energy expenditure. Increasing physical activity is an efficient way to raise energy expenditure because, for reasons not clearly understood, total daily expenditure is usually increased to a greater degree than could be predicted from the energy cost of the additional physical activity itself. In addition, most available studies indicate that people do not compensate by being less active at other times. Increased physical exercise that is maintained over several months usually helps body weight loss, although not systematically. However, even in the absence of body weight changes, body composition may be modified by losing fat and gaining fat-free mass (depending on the type of exercise). Future research should examine the elusive energy expenditure component that is not accounted for by the energy cost of exercise and explore the possible gender differences in body weight response to exercise. Additional research should also determine thresholds of physical activity that would prevent the gradual increase in body weight during adulthood and that would help the post-obese maintain weight loss in the long term.

In addition to total energy expenditure, interactions between dietary fat/carbohydrate ratio and physical activity may play a crucial role in the susceptibility to obesity, remarked Prof. A. Astrup (Royal Veterinary \& Agricultural University, DK). Fat possesses a lower satiating power than does carbohydrate and protein, and a diet low in fat therefore decreases energy intake. Dietary carbohydrate promotes its own oxidation by an insulin-mediated stimulation of glucose oxidation. In contrast, high fat meals do not result in an immediate increase in fat oxidation, which could lead to episodic bouts of fat storage. Observational and intervention studies indicate that low-fat diets eaten ad libitum result in a weight loss that seems to be body weight dependent, so that overweight and obese subjects lose more than normal weight subjects. Experimental studies have shown that energy balance can be maintained at a higher level of dietary fat intake when physical activity level is also high. Therefore, physical activity increases the tolerance to dietary fat. Genes are being discovered that control various aspects of energy balance and expenditure in experimental animals. However, their involvement in human obesity is far from understood. Future research should address the genetics of mechanisms responsible for the balance of fuels (fat and carbohydrate) used and relate these to why certain susceptible individuals passively over-consume food and gain weight.

Dr. M. Fogelholm (University of Helsinki, SF) recognised that concern has been expressed that increased physical activity, particularly at the athletic level, might result in increased micronutrient requirements because of higher turnover and excretion rates. The evidence indicates that micronutrient intake increases with the additional energy intake required for physical activity, although at a slightly lower nutrient density. The available indices of micronutrient status do not support the belief that micronutrient status is compromised in highly trained athletes, even without the use of dietary supplements. Hence, there are no reasons to believe that moderately active people following a normal, mixed Western diet are at risk of compromised micronutrient status.

According to Dr. F. Bellisle (Hôtel Dieu de Paris, F), food choices and diet composition have been studied less often than energy intake in subjects with varying levels of physical activity. In most of the available short term studies, there is no evidence that an acute bout of exercise increases appetite. In longer-term studies, active people often report increased carbohydrate intake, and decreased saturated fat intake (however, opposite results have also been published). Several potent factors determine food choice and intake in active people as well as in sedentary people: life-long habits, preferences, aversions, social context, cognition, psychological traits (such as health concerns and chronic dietary restraint) and energy density of 
available foods. Such factors can have more influence on food intake than do the immediate signals induced by a bout of exercise. In most studies, carried out in laboratories or in the field, these factors do have more influence, no matter how long food intake is studied.

Dr. J.J. Grimm (Lausanne University Hospital, CH) discussed the implications of diet/physical activity interactions for glucose-insulin dynamics. Type II diabetes is a public health issue in developed and developing countries and insulin resistance syndrome is the main risk factor for Type II diabetes. There is evidence that exercise interventions can contribute to reduced abdominal fat, improved blood pressure levels, improved glucose tolerance and reduced insulin resistance thus reducing risk of developing Type II diabetes. It is important to note that exercise interventions are often accompanied by favourable dietary changes, but there is no evidence showing a reinforcing effect of diet on the exercise intervention. Regular endurance exercise has the potential to decrease insulin resistance.

The influence of physical exercise in lipoprotein (blood lipid) metabolism was the topic presented by Prof. A. Hardman (Loughborough University, UK). People who are physically active experience a lower incidence of cardiovascular disease and exhibit lipoprotein profiles consistent with this lower risk. The main beneficial effects of exercise are to increase high density lipoprotein (HDL) cholesterol and to decrease both fasting and postprandial (post-meal) levels of plasma triacylglycerol (TAG). This effect is evidenced even for regular, moderate exercise such as walking. Research indicates that high carbohydrate diets reduce HDL-cholesterol and that people who become more active increase dietary carbohydrates. However, physically active individuals show higher levels of HDLcholesterol despite an increase in dietary carbohydrate and decrease in fat.

The evidence that blood pressure is an important risk factor for cardiovascular disease was reviewed by Dr. B. Margetts (University of Southampton, UK). There is good evidence to support the opinion that lowering high blood pressure contributes to reduced mortality. Controlled trials support the view that dietary changes - including a higher consumption of fruit, vegetables and whole grain cereals- and $30 \mathrm{~min}$ regular, moderate physical activity (lifestyle interventions) can effectively bring about a reduction of $3-5 \mathrm{~mm} \mathrm{Hg}$ in blood pressure. Trials in a Primary Care setting have, thus far, lacked clarity with regard to compliance and possible bias leading some to conclude that this kind of intervention does not work. This field warrants further investigation. Primary Care staff need better training and support in order to be able to conduct more reliable studies.
Dr. R. Rauramaa (University of Kuopio, SF) discussed the implications for haemostatic (bood clotting) factors. Regular moderate intensity physical activity as well as a diet rich in omega- 6 fatty acids decrease platelet aggregability and consequently the tendency for blood to clot. Other effects of physical activity and diet remain less clear. There are few controlled clinical trials on interactions between physical activity and diet on haemostatic factors, but it can be hypothesised that the two will interact to have a beneficial effect. More research is needed in this respect.

The prevention of osteoporosis should start early in life, by optimising peak bone mass, and bone mass should be protected throughout life, remarked Dr. F. Branca (National Institute of Nutrition, I). Diet and physical activity account for between $20 \%$ and $40 \%$ of the variability in bone mass. The geometric properties of the bone are determined by habitual loads and the intensity of the load is more important than the duration of the exposure. Weight bearing activities such as walking, running, jumping and resistance training have a greater enhancing effect on bone mineral density than do non-weight bearing activities. Immobilisation or interruption of training causes bone loss. Excess exercise can be a problem in elite athletes whether male or female but there is no evidence of adverse effects of moderate exercise on children's growth. Calcium and other mineral requirements have to be met in order to build bone.

There is good evidence that being overweight is a risk factor for cancer at many sites, particularly for hormone-related cancers, stated Dr. M. Hill (Wexham Park Hospital, UK). According to a recent report from the UK Committee on Medical Aspects of Food Policy ${ }^{5}$ there is little evidence for an association between cancer and excess energy intake. It seems more evident that a sedentary lifestyle, leading to overweight, is the potential cause of an increased cancer risk. Some studies suggest that physical activity has a protective role against cancer of the colon, breast and prostate; recreational activity seems to be more important than occupational activity. Further research is required to identify which type of physical activity is most beneficial and to clarify the underlying mechanisms.

Dr. D. Benton (University of Wales Swansea, UK) addressed the relationship between diet, mood and well-being. Several types of depressive disorders have been associated with an increased intake of high carbohydrate foods but these findings are often difficult to interpret. In normal individuals a higher intake of carbohydrate over several days is associated with better mood, although the mechanism remains unclear. Also chocolate, one of the most craved food items, has mood elevating properties, possibly by stimulating endorphin release in the brain. Mood changes may 
precede other sub-clinical signs of nutritional deficiencies as in the case of thiamin deficiency. Perhaps this is why some studies suggest that supplementation contributes to improved mood. For example, folate deficiency in elderly people has been associated with depression and iron deficiency anaemia can be associated with apathy.

Dr. K. Fox (University of Exeter, UK) highlighted the fact that mental health problems seem to be on the increase. There is growing evidence demonstrating that exercise can be effective in improving the mental wellbeing of the general public, largely through improved mood and self-perceptions. He presented evidence that exercise is effective as a potential preventative measure or a therapy for clinical or sub-clinical depression or anxiety. Regular physical activity may also enhance self-esteem and improve sleep, thus contributing to mental well-being.

Physical activity affects many systems of the body and provides numerous health benefits for adults. There is substantial interest in beginning the prevention of adult diseases during the first two decades of life through regular physical activity. However, there is controversy regarding the effects of physical activity in youth on chronic disease in adulthood because longterm studies are lacking. Moreover, although there is a considerable amount of data on physical fitness in young people, few studies have assessed the interaction between life-style and disease risk factors from an early age onward over longer periods of time, remarked Prof. W. van Mechelen (Free University, NL). One such prospective study is the Amsterdam Growth and Health Study (AGHS). Among the major outcomes of the AGHS, it was concluded that: 1. There is no tracking in physical activity nor in physical inactivity (i.e. individuals change their life-style habits over time; 2. There is better maintenance of bone health when various weight-bearing activities (such as running, cycling, jumping) are performed during the growing years, than simply when calcium intake is above recommended dietary allowance; 3. From a public health viewpoint, adolescent physical inactivity is as detrimental as excessive smoking.

The influence of diet and physical activity in older adults was discussed by Prof. A. Dontas (Hellenic Association of Gerontology-Geriatrics, GR). Interactions between diet and physical activity are important in old age, as diets are often deficient and activity can be poor because of disability and lack of energy. Rehabilitation measures with resistance exercise improve muscle strength and, in association with nutritional supplements ( $17 \%$ protein), can lead to increased lean body mass and restore physical independence in the elderly. Even in very old, frail individuals, this kind of prevention is effective and has been associated with decreased overall mortality rate.

\section{Research Needs, Developments and Conclusions}

One of the main topics addressed in the final discussion of the workshop was the need for further research on interactions and potential synergy between diet and physical activity as an effective means for the prevention and treatment of obesity, cardiovascular diseases and cancer. Recent other publications have also addressed this issue $e^{6,7,8}$.

The importance of good scientific evidence on which to base clear messages to the public was noted. A major gap in current knowledge is the lack of standardized information on patterns of physical activity in the population which can be used as baseline data against which to measure future change. It was noted that people tend to over-report their level of physical activity and under-report their energy intake. To address the lack of data on physical activity, Prof. M. Sjöström informed about a new tool, an International Physical Activity Questionnaire (IPAQ), that is being validated for young and adult people in different countries. Recommendations were also made to gather comparable pan-European data on food. nutrient intake and nutritional status.

Effective population-based strategies in Europe in the field of nutrition and physical activity, i.e. public health nutrition, require people that are competent and properly trained. Dr. A. Yngve (Karolinska Institutet, S) described how common standards of training should be set and monitored. We can hope for increased mobility and networking between European nutrition and physical activity professionals through the European Commission's support to post-graduate training (Master's Programme). This will result in a new generation of professionals who have an integrated view of public health nutrition.

The workshop supported the conclusions of two previous recent consensus conferences ${ }^{9,10}$ which advised people, both adults and young people, to participate in at least $30 \mathrm{~min}$ of moderate physical activity on most, preferably all, days of the week. However, advice needs to be flexible and a range of different options should be offered, taking occupational and recreational physical activity patterns into account.

The difficulties of enhancing physical activity patterns in modern society were also discussed. Health promotion strategies, including behavioral interventions aimed at modifying dietary habits and physical activity patterns, should start from the early stages of life. Developmental stages of children need to be considered when designing appropriate messages. Some experiences of community programs involving elderly people suggest that exploring appropriate strategies and motivations for active living in this age group can also be rewarding. 
Discussions underlined that when people become more active, their dietary habits change to a more balanced and varied diet. In order to achieve the goal of getting people to be moderately active for 30 min daily, it was agreed that a great deal more effort was needed to bring this topic to the attention of the public and, particularly, to the attention of policy makers.

\section{References}

1 Murray CJL, Lopez AD. The Global Burden of Disease. Boston: Harvard School of Public Health, The World Health Organization and the World Bank/Harvard University Press, 1996.

2 Bouchard C, Shepard RJ, Stephens T. Physical Activity, Fitness and Health; International Proceedings and Consensus Statement. Champaign, IL: Human Kinetics Publishers Inc., 1994.

3 Strain JJ (ed.). Institute of European Food Studies: Pan-Eu survey of consumer attitudes to food, nutrition and health. Eur J Clin Nutr 1997; 51: Supplement 2.

4 Flynn A (ed). Pan EU survey of consumer attitudes to physical activity, body weight and health. Public Health Nutrition 1999; 2: Supplement 1(A).

5 Department of Health, RHSS 48: Nutritional Aspects of the Development of Cancer. London: The Stationary Office, 1998.

6 Department of Health, RHSS 46: Nutritional Aspects of Cardiovascular Disease. London: The Stationary Office, 1994.

7 WHO. Obesity. Preventing and managing the global epidemic. Geneva: WHO, 1998.

8 Food, Nutrition and the Prevention of Cancer: a global perspective. Washington, DC: World Cancer Research Fund/ American Institute for Cancer Research, 1997.

9 Pate R, Pratt M, Blair S, Haskell W, Macera CA, et al. Physical Activity and Public Health: A recommendation from the Centers for Disease Control and Prevention and the American College of Sports Medicine. JAMA 1995; 273: 402-7.

10 Young and Active? Young people and health-enhancing physical activity - evidence and implications. London: Health Education Authority, 1998. 